\title{
Exploring Images: A Haptic Mouse Approach
}

\author{
http://dx.doi.org/10.3991/ijoe.v8iS1.1889
}

\author{
J. Wolfer \\ Indiana University South Bend, South Bend, USA
}

\begin{abstract}
Exposing students to touch-enabled computing typ- ically requires the use of expensive Haptic robots. This exhibit demonstrates the adaptation of a commercial, haptically-enabled mouse. Using a customized Linux device driver and a Python imaging script, we allow users to "virtually touch" images. Specific examples include exploring the Mona Lisa, digital mammograms, and "touch-traversal" of a maze.
\end{abstract}

Index Terms - Haptic interfaces, Image processing, Human computer interaction.

\section{INTRODUCTION}

Exposing students to touch-enabled computing typically requires the use of expensive Haptic robots, such as the Sensable Phantom series [1]. Other examples of haptics can be found in Salisbury et. al. [2], who provides a description of haptics ranging from force-enabled mice to haptic exoskeleton interfaces. A haptic mouse interface is also described by Takasaki et.al. [3]. Their investigation indicated that a tactile display mouse could provide surface textural information, but that shape classification is more challenging.

\section{HAPTIC MOUSE}

This exhibit features an inexpensive tactile feedback mouse, the Logitech Ifeel mouse. While no longer in active production, the mouse is still available, and it provides an effective, inexpensive, platform for introducing haptics. From outward appearance this is just another USB mouse, however, in addition to conventional interactive functions this mouse contains a programmable tactile generator which creates sensations reminiscent of modulated cell-phone vibrators. Specifically, the mouse accepts three parameters to control feedback: vibration intensity, delay between vibration events, and the number of times to repeat.

\section{SOFTWARE}

To ensure that the software could be distributed to students we elected to adapt existing Linux ifeel mouse drivers [4] and embed them into a live Knoppix Linux [5] distribution. For the image processing we created an interface that would allow us to work with a variety of images, generate appropriate force feedback events, and be flexible enough to adapt to classroom and research applications. The software is scripted using the Python programming language and includes functions for reading and displaying an image, determining the current location of the mouse, and generating tactile feedback commands.

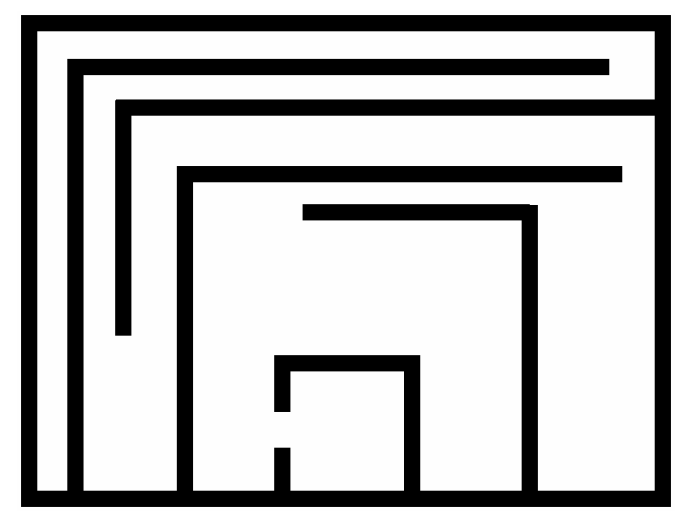

Figure 1. Haptic Maze

\section{DEMONSTRATIONS}

For this exhibit three different images will be presented, each with a custom version of the software to explore specific aspects of the underlying pixels.

\section{A. Haptic Maze Traversal}

This example was designed to give students a "roboteye" view of a maze by restricting feedback to touching the wall. Figure 1 shows a replica of one of our computer mazes (dark/light inverted for printing). In this case the tactile mouse is directed to vibrate intensely when it encounters a barrier, and is totally still otherwise, simulating a proximity switch on a robot. Specifically, the image intensity ( 0 or 255 in this binary image) provides the intensity, with empirically determined delay and repeat values of five and ten respectively.

\section{B. Mona Lisa}

The Mona Lisa, shown in Figure 2, is used to explore more subtle image textures. As the mouse traverses the image it is modulated with brighter pixels generating more vigorous sensations and darker pixels providing a more sedate sensation. In this case the pixel value under the mouse cursor provides the vibration intensity, with a single repeat after a $15 \mathrm{msec}$ delay.

\section{Mammograms}

The mammogram exhibit demonstrates a potentially practical application. Specifically, we show an application that implements an additional information channel. Figure 3 shows a digital mammogram from the Digital Database for Screening Mammography [6], which contains one malignant lesion. 


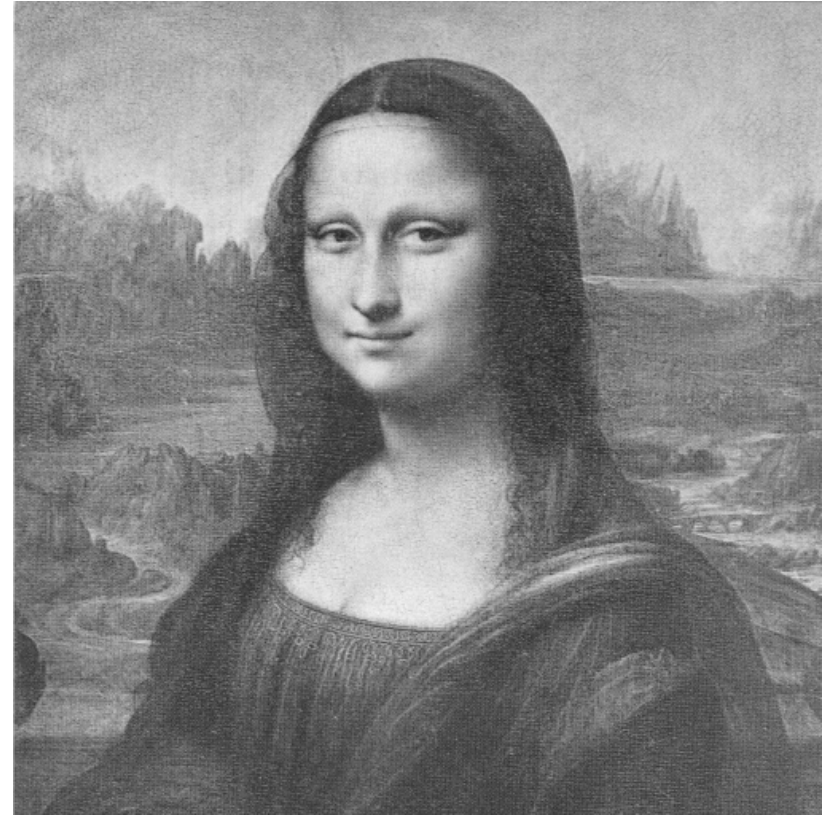

Figure 2. Mona Lisa

In this case all three mouse parameters are modulated as a function of the underlying image pixel value to exaggerate the response to brighter pixels typical of those in a region containing a lesion. Specifically, the vibration intensity is restricted an interval between 200 and 255 (as opposed to 0 - 255) as a linear function of pixel intensity under the mouse pointer. This produces a vigorous baseline response. The delay between repeat cycles is also modulated, with brighter pixels generating shorter delays and more repeat cycles.

\section{CONCLUSION}

The exhibit proposed here demonstrates the use of an inexpensive, touch-enabled, mouse to render image data haptically. This infrastructure provides a cost-effective first introduction to students while providing an application platform for further studies.

\section{REFERENCES}

[1] Sensable, "Phantom Omni Haptic Device," http://www.sensable.com.

[2] I. S. Salisbury, F. Conti, and F. Barbagli, "Haptic Rendering: Introductory Concepts,” in IEEE Computer Graphics and Applications, March, 2004.

[3] M. Takasaki, T. Nara, and T. Mizuno, "Haptic expression of images using a surface acoustic wave tactile display mouse", IEEE International Symposium on Industrial Electronics, 2003.

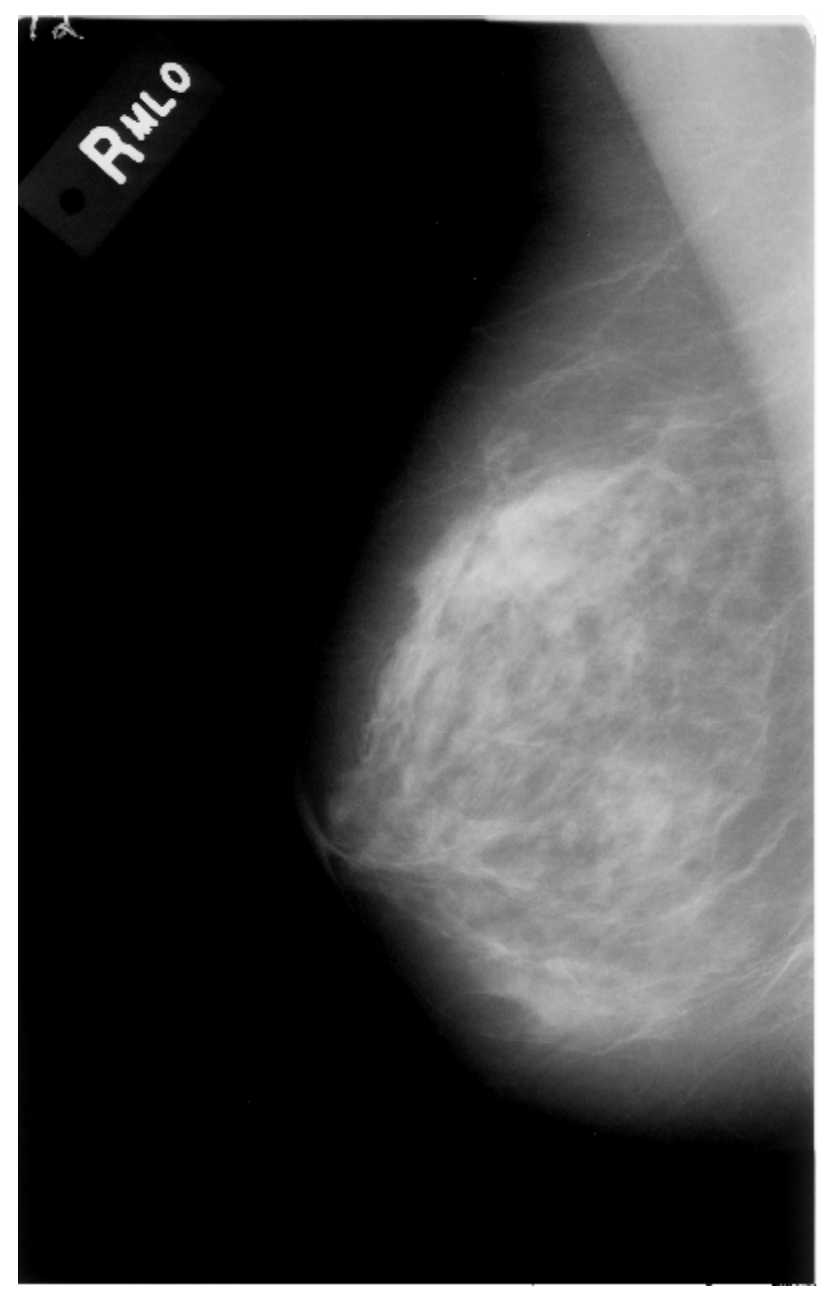

Figure 3. Mammograms

[4] Sourceforge, "ifeel mouse driver, http://www.sourceforge.net/ projects/tactile.

[5] “Knoppix Linux,” http://www.knoppix.net

[6] U. of South Florida, "Digital Database for Screening Mammography,” http://marathon.csee.usf.edu/Mammography/Database.html

\section{AUTHORS}

J. Wolfer is with the Indiana University South Bend Department of Computer Science, South Bend, IN, USA (email: jwolfer@iusb.edu)

This work is a description of a demonstration given during $1^{\text {st }}$ Experiment@ International Conference, 17/18 November 2011 in Lisbon, Portugal. It was supported by Calouste Gulbenkian Foundation, Lisbon, Portugal. Received 01 December 2011. Published as remsubmitted by the author 20 January 2012. 